\title{
Modelling and simulation of magnesium antimonide based thermoelectric generator
}

\author{
Md. Nazibul Hasan ${ }^{1}$, Herlina Abdul Rahim ${ }^{2}$, Mohd Ashraf Ahmad ${ }^{3}$, Mohamed Sultan Mohamed Ali ${ }^{4}$ \\ ${ }_{1,2,4}$ School of Electrical Engineering, Universiti Teknologi Malaysia, Malaysia \\ ${ }^{3}$ Faculty of Electrical and Electronics Engineering, Universiti Malaysia Pahang, Malaysia
}

\begin{tabular}{l} 
Article Info \\
\hline Article history: \\
Received Jan 30, 2020 \\
Revised Mar 6, 2020 \\
Accepted Mar 22, 2020 \\
\hline Keywords: \\
Finite element analysis \\
Power efficiency factor \\
Thermocouple \\
Thermoelectric generator
\end{tabular}

\section{Corresponding Author:}

Mohamed Sultan Mohamed Ali, School of Electrical Engineering, Universiti Teknologi Malaysia, 81310 UTM Johor Bahru, Johor, Malaysia. Email: sultan_ali@fke.utm.my

\begin{abstract}
This paper presents the modelling and simulation of a $\pi$-shaped $\mathrm{Mg}_{3} \mathrm{Sb}_{2}$ based thermoelectric generator. The performance of the proposed thermoelectric generator is evaluated with finite element analysis. A number of thermocouples were varied for high output power and power efficiency factor. Based on the analysis, we demonstrated that enhancement of the temperature gradient and the number of thermocouples are beneficial for high output power and power efficiency factor of $\mathrm{Mg}_{3} \mathrm{Sb}_{2}$ based thermoelectric generator. A high output power and power efficiency factor of $8.89 \mathrm{~mW}$ and $3.47 \mathrm{mWmm}^{-2} \mathrm{~K}^{-2}$ were obtained at a temperature gradient of $500 \mathrm{~K}$ across the hot and cold side for four $\mathrm{Mg}_{3} \mathrm{Sb}_{2}$ based thermocouples, respectively. The obtained results show that the developed device could be used to drive portable electronic devices.
\end{abstract}

Copyright $($ C 2020 Institute of Advanced Engineering and Science. All rights reserved.

\section{INTRODUCTION}

With the rapid growth of industrialization, the need for electricity in everyday life is enhanced. Due to the deficit of fossil fuel, natural gas, and coal, the alternative source of energy is one of the major challenges in the $21^{\text {st }}$ century. Besides, the emission of carbon while fossil fu el and coal burnt has a great effect to accelerate global warming [1]. Thus reduction of carbon emissions has become a global priority owing to the serious effect on climate change. To counter this issue, significant effort has been given to developed green energy harvesters. Green energy harvesters transduce various forms of energy such as sunlight, mechanical vibrations, ocean waves, and human body heat into electrical energy while maintaining a green energy environment [2-3]. A thermoelectric generator (TEG) is a kind of energy harvester that transmutes heat energy to electrical energy employing the principle of the Seebeck effect [4-5]. It has several features such as its high reliability and durability at low cost, no maintenance required, and direct conversion with no intermediate energy conversion process [6-7]. Besides, it has the potential to enhanced the longevity of an electrical device while maintaining both emissions and noise free operation i.e. provides clean energy by reducing greenhouse gas and carbon emissions [8]. Thus, this device has been widely used to power portable electronic devices such as glucose monitoring device [9], electroencephalography (EEG) [10], accelerometer [11], sweat conductivity monitoring [12], pressure-temperature Sensor [13], and human motion monitoring [14], whose power requirement is in the range of $\mathrm{mW}$ to $\mu \mathrm{W}$.

To design an efficient TEG, two factors must be taken into considerations such as thermoelectric (TE) materials and heat loss reduction. Among TE materials, bismuth telluride $\left(\mathrm{Bi}_{2} \mathrm{Te}_{3}\right)$ is widely used for TE power generations due to its high efficiency at near room temperature. For instance, Chen $e t$ al. fabricated a TEG with $\mathrm{Bi}_{2} \mathrm{Te}_{3}$ and $\mathrm{Bi}_{0.5} \mathrm{Sb}_{1.5} \mathrm{Te}_{3}$ as $n$-type and $p$-type TE legs, and obtained maximum output power $\left(P_{\text {out }}\right)$ 
of $127 \mathrm{nW}$ at a temperature difference of $32.5 \mathrm{~K}$ [15]. Nour et al. modelled a TEG with $\mathrm{Bi}_{2} \mathrm{Te}_{3}$ and showed a $P_{\text {out }}$ of $1.5 \mathrm{~W}$ for hot and cold side temperatures of $671 \mathrm{~K}$ and $354 \mathrm{~K}$, respectively [16]. Kong et al. developed a wearable TEG employing flexible $\mathrm{Bi}_{2} \mathrm{Te}_{3}$ films and achieved open circuit voltage and $P_{\text {out }}$ of $48.9 \mathrm{mV}$ and $693.5 \mathrm{nW}$, respectively [17]. Besides, lead telluride ( $\mathrm{PbTe}$ ) has been widely studied for medium range temperature TE applications i.e. in the range in between (450-850) K. For example, Mei et al. modelled a segmented TEG with $\mathrm{PbTe}_{-} \mathrm{Bi}_{2} \mathrm{Te}_{3}$ for enhanced device efficiency [18]. Wang et al. designed TEG with PbTe legs, and obtained a $P_{\text {out }}$ of $7.6 \mathrm{~W}$ at a temperature difference of 500K [19]. Nevertheless, toxicity and exiguous quantity of lead $(\mathrm{Pb})$ and tellurium $(\mathrm{Te})$ presented in these materials make them adverse for TE applications. Alternatively, magnesium antimonide $\left(\mathrm{Mg}_{3} \mathrm{Sb}_{2}\right)$, a non-toxic and abundant in nature, has been emerged as promising thermoelectric material owing to its excellent TE properties such as its high $S$ which is around $300 \mu \mathrm{VK}^{-1}$ and low thermal conductivity <1 [20]. Typically, $\mathrm{Mg}_{3} \mathrm{Sb}_{2}$ behaves as a $p$-type material due to the intrinsic vacancy of $\mathrm{Mg}$ and thus exhibits low $\sigma$ and overall $Z T$ value of 0.94 [21-22]. Nevertheless, using proper dopants its behaviour has been transformed into the $n$-type material with the highest $Z T$ value up to 1.85 [23].

This paper presents a 3D (dimensional) modelling and simulations of a $\pi$-shaped TEG with $p$-type and $n$-type $\mathrm{Mg}_{3} \mathrm{Sb}_{2}$ based TE legs. The heat distribution through the $p$-type and $n$-type $\mathrm{Mg}_{3} \mathrm{Sb}_{2}$ based TE legs are analyzed. Besides, the generated thermoelectric voltage, maximum output power, and thermoelectric power efficiency factor are calculated and analyzed using finite element analysis. This paper is organized as follows: the design and working principle of TEG are discussed in Section 2. Section 3 presents the finite element analysis and governing equations for the TEG operation. Section 4 presents the key results and analysis, and finally, the paper is concluded in Section 5.

\section{DESIGN AND WORKING PRINCIPLE}

The schematic diagram of a $\pi$-shaped TEG with thermocouples and its equivalent circuit is presented in Figure 1. It constitutes two main parts such as TE legs and its connecting electrodes. Herein, both the TE legs consists of the $p$-type and $n$-type $\mathrm{Mg}_{3} \mathrm{Sb}_{2}$. These legs are electrically connected in series and thermally in parallel. The geometrical dimensions of one thermocouple are shown in Table 1. Whenever a temperature gradient, $\Delta T$, is applied in between two TE legs, the majority charge carries presented at each TE leg tends to diffuse from higher concentration to lower concentration, as a consequence, an electric potential will initiate across the TE legs [24]. The heat flux $(Q)$ is applied across hot to the cold side and electrical current ( $I$ ) flows from $n$-type to the $p$-type material due to $\Delta T$, as depicted in Figure 1(a). The performance of a TEG is defined by a dimensionless figure of merit $(Z T)$, which is defined as:

$$
Z T=\frac{s^{2} \sigma}{k}
$$

where $S, \sigma, k$ is the Seebeck coefficient, electrical conductivity, total thermal conductivity, respectively. The equation signifies that a TEG should constitute a high $S$ to enhance the conversion of heat into electrical power, a high $\sigma$ to reduce Joule heating [25], high power factor $\left(P F=S^{2} \sigma\right)$, low thermal conductivity $(k)$ to hinder thermal shorting [26], and should maintain a large $\Delta T$ in between the hot and cold sides [27]. Figure 1(b) shows the equivalent circuit model where TEG is modelled as a voltage source, $V_{T E}$, in series with a resistor and which is defined as [28-29]:

$$
V_{T E}=n S_{n p}\left(T_{H}-T_{C}\right)=n S_{n p} \Delta T
$$

Table 1. Geometrical dimensions of a thermocouple

\begin{tabular}{cccc}
\hline Material & Length $(\mathrm{mm})$ & Thickness $(\mathrm{mm})$ & Width $(\mathrm{mm})$ \\
\hline$n$-type and $p$-type leg & 5 & 0.8 & 0.8 \\
Top side electrode & 0.3 & 0.8 & 2.4 \\
Bottom side electrode & 0.3 & 0.8 & 1.6 \\
\hline
\end{tabular}

where $n$ is the number of thermocouples, $S_{n p}$ is the relative Seebeck coefficient of $p$-type and $n$-type TE legs, and $T_{H}$ and $T_{C}$ are the thermocouple temperatures of the hot and cold side. The TE equivalent resistance, $R_{T E}$, is the total resistance offered by TE legs $\left(r_{T}\right)$ and the electrodes $\left(r_{e}\right)$, which is defined as [30]:

$$
R_{T E}=n\left(r_{T}+r_{e}\right)=n\left[\left\{\left(\frac{1}{\sigma_{p}}+\frac{1}{\sigma_{n}}\right) \frac{l_{T E}}{t_{T E} \times w_{T E}}\right\}+\frac{l_{e}}{\sigma_{e}\left(t_{e} \times w_{e}\right)}\right]
$$


where $\sigma_{p}, \sigma_{n}$, and $\sigma_{e}$, are the conductivity of $p$-type TE leg, $n$-type TE leg, and electrode, respectively, $l_{T E}$ and $l_{e}$ are the length of TE legs and electrode, respectively, and $t_{T E}, w_{T E}, t_{e}$, and $w_{e}$ are the thickness of TE legs, width of TE legs, thickness of the the electrode, and width of the electrode, respectively. It should be noted that the $r_{T}$ is temperature dependent and it decreases with elevated temperature. Once the $V_{T E}$ and $R_{T E}$ are estimated, the current $(I)$ through the TEG can be calculated using the following expression:

$$
I=\frac{V_{T E}}{R_{T E}}
$$

(a)
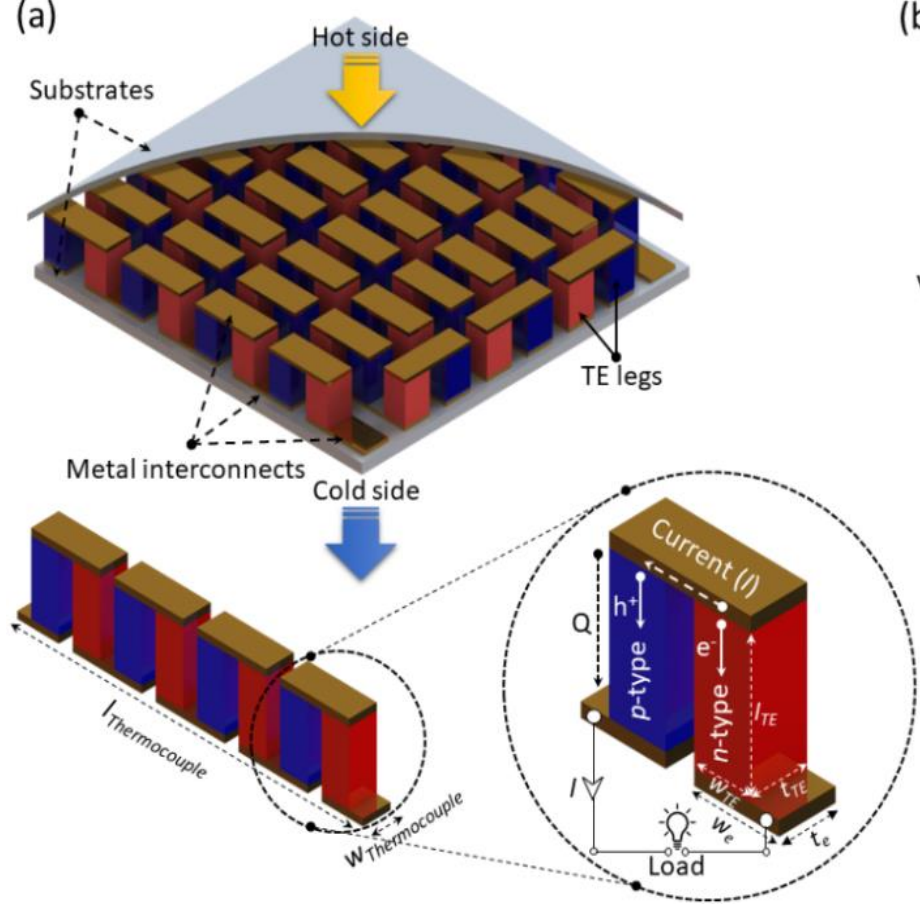

(b)

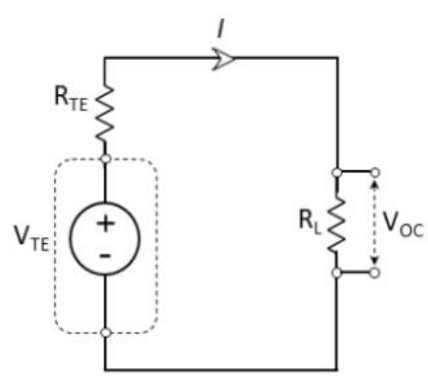

Figure 1. (a) Schematic diagram of a TEG module with thermocouples and (b) equivalent circuit of a thermocouple

The $P_{\text {out }}$ and thermoelectric power efficiency factor $(\phi)$ are defined by the following expressions [28, 30]:

$$
P_{\text {out }}=\frac{V_{T E}}{4 R_{T E}}
$$

and

$$
\phi=\frac{P_{\text {out }}}{\left(l_{\text {Thermocouple }} \times W_{\text {Thermocouple }}\right) \times \Delta T^{2}}=\frac{P_{D}}{\Delta T^{2}}
$$

where $l_{\text {Thermocouple }}$ and $w_{\text {Thermocouple }}$ are the length and width of the complete TEG, respectively, and $P_{D}$ defines the output power of the TEG. All these parameters are used for analysis, calculate and plot the $V_{T E}, P_{o u t}$, and $\phi$ in the following section.

\section{FINITE ELEMENT ANALYSIS}

In this work, COMSOL Multiphysics® was used to do the numerical simulation of a $3 \mathrm{D}$ model of the TEG. Heat transfer and AC/DC modules were used throughout the simulation process. Both these modules are used to build the thermoelectric effect and electromagnetic heating of TEGs depending on material properties and geometrical dimensions. It was assumed that the $T_{H}$ was $300 \mathrm{~K}$, convective heat flux, $Q$, was $40 \mathrm{Wm}^{-2} \mathrm{~K}^{-1}$ which is suitable for real time environment, and there in no heat loss during simulation, nevertheless, heat losses are there to the surroundings in the practical case. 
In the heat transfer module, whenever $\Delta T$ is applied across the TE materials, the heat flow equation in the thermoelectric analysis (7) and the relationship between heat flux, and current density of TE materials i.e. the energy conservation for a solid domain that includes Joule heating (8) can be obtained, which are defined as follows [31]:

$$
\left(\rho_{d} C_{p} \frac{\partial T}{\partial t}+\Delta . Q\right)=Q_{g}
$$

and

$$
\Delta(k . \Delta T)-T . J \cdot\left(\frac{\partial S}{\partial T}\right)+\rho_{T E} \cdot J=0
$$

where $\rho_{d}, C_{p}, Q, Q_{g}, k, J$, and $\rho_{T E}$ defines the density, specific heat capacity, heat flux, heat generation rate per unit volume, thermal conductivity, current density, and resistivity of the thermoelectric module, respectively. The heat flux, $Q$, in TEG module is defined as the following (9) [32]:

$$
Q=-(k \Delta T)
$$

Whenever the TEG module reaches a steady state condition, the electric charge and temperature distributions are stable. So we get the following equations in AC/DC modules [33]:

$$
\begin{aligned}
& \Delta . J=Q_{j} \\
& E=-\Delta V \\
& J=\left(\sigma E+J_{e}\right)
\end{aligned}
$$

where $J, J_{e}, Q_{j}$, and $E$ defines the current density, external current density, current source, and electrical field, respectively. All the (10-12) define the continuity of current, the electrical potential, and current density, respectively.

\section{RESULTS AND ANALYSIS}

After employing the governing equations, the simulation results and the performances of the $\mathrm{Mg}_{3} \mathrm{Sb}_{2}$ based TEG are analyzed in this section. The temperature distribution through $p$-type and $n$-type TE legs and the effect of varying the number of thermocouples on $V_{T E}, P_{\text {out }}$, and $\phi$ are observed and calculated by using COMSOL Multiphysics ${ }^{\circledR}$. The heat distribution of a 3D thermocouple through the $p$-type and $n$-type $\mathrm{Mg}_{3} \mathrm{Sb}_{2}$ based TE legs is shown in Figure 2. The $T_{C}$ and $T_{H}$ of the thermocouple are set to $300 \mathrm{~K}$ and $800 \mathrm{~K}$, respectively, and $Q$ of $40 \mathrm{Wm}^{-2} \mathrm{~K}^{-1}$ is applied in order to observe the heat transfer from hot to the cold side as shown in Figure 2(a). It can be noted that the heat distribution through the length of $n$-type TE leg is almost linear, nevertheless, the graph is deviated for $p$-type TE leg in between the length of $\sim(3-4.5)$ mm as shown in Figure 2(b). It's due to the $k$ value which is slightly higher for $n$-type than $p$-type $\mathrm{Mg}_{3} \mathrm{Sb}_{2}$ based TE legs.

After attaining the temperature distribution through the TE legs, $V_{T E}$ is investigated. Figure 3(a) shows the 3D simulation results of a thermocouple with the direction of heat flux form hot to the cold side and its generated $V_{T E}$ for with $T_{C}$ and $T_{H}$ of $300 \mathrm{~K}$ and $800 \mathrm{~K}$, respectively. The cold side of the thermocouple is grounded i.e. $0 \mathrm{~V}$ to develop $V_{T E}$ when the heat is supplied at the $T_{H}$. As the $\Delta T$ in between $\mathrm{Mg}_{3} \mathrm{Sb}_{2}$ based TE leg increases, the $V_{T E}$ also increases since $V_{T E}$ is directly proportional to $\Delta T$ according to (2). Besides this equation shows the effect of enhancing the number thermocouples on the generated $V_{T E}$ as shown in Figure 3(b). As the number thermocouple increases, the maximum amount of $\Delta T$ dissipates through the thermocouples and as a result in the enhancement of $V_{T E}$. A maximum $V_{T E}$ of $727 \mathrm{mV}$ is obtained at $\Delta T=500 \mathrm{~K}$ for four $n$-type and $p$-type $\mathrm{Mg}_{3} \mathrm{Sb}_{2}$ based thermocouples.

The $P_{\text {out }}$ and $\phi$ of $\mathrm{Mg}_{3} \mathrm{Sb}_{2}$ based thermocouples are plotted graphically versus $\Delta T$ in Figure 3(c) and 3(d). It can be noted from the plot that both $P_{\text {out }}$ and $\phi$ increases exponentially while enhancing the $\Delta T$ and number of thermocouples. Both these parameters depend on the $\sigma$ of the $p$-type and $n$-type $\mathrm{Mg}_{3} \mathrm{Sb}_{2}$ based TE legs. As a result, $\sigma$ of the $p$-type and $n$-type $\mathrm{Mg}_{3} \mathrm{Sb}_{2}$ based TE legs increases with increasing temperature and the overall resistance of the thermocouple decreases according to (3). Thus, both $P_{\text {out }}$ and $\phi$ are increasing exponentially as $P_{\text {out }}$ is inversely proportional to overall resistance and $\phi$ is directly proportional to $P_{\text {out }}$ according to (5) and (6), respectively. A maximum $P_{\text {out }}$ and $\phi$ of $8880 \mu \mathrm{W}$ and $3468.7 \mu \mathrm{Wmm}^{-2} \mathrm{~K}^{-2}$ is obtained at $\Delta T=500 \mathrm{~K}$ for four $n$-type and $p$-type $\mathrm{Mg}_{3} \mathrm{Sb}_{2}$ based thermocouples, respectively. 
(a)

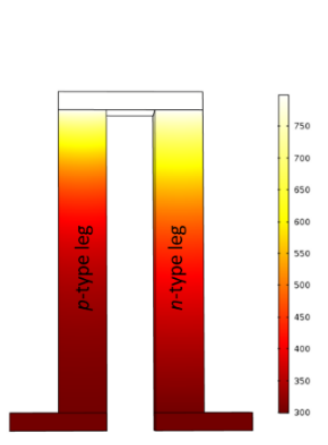

(b)

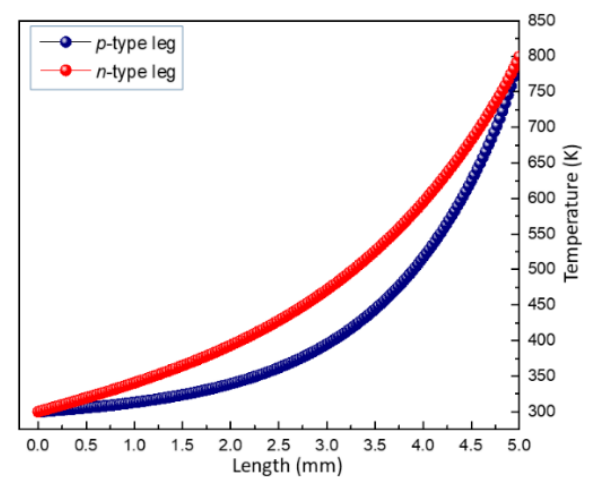

Figure 2. Simulation results showing (a) 3D heat distribution profile through one thermocouple and (b) within the TE legs

(a)

(c)
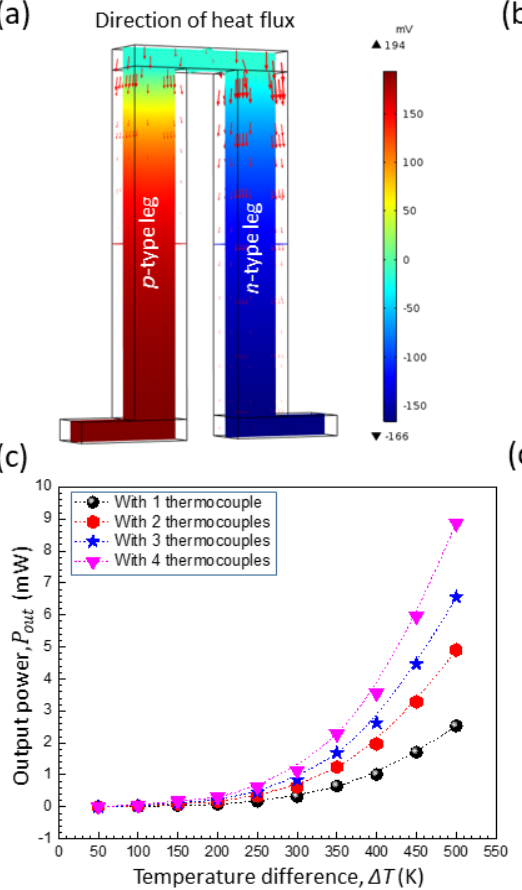

(b)

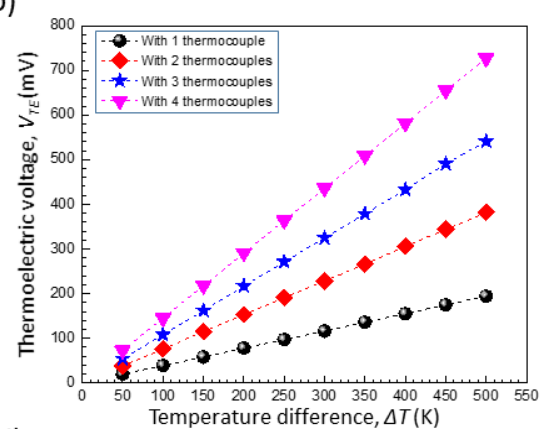

(d)

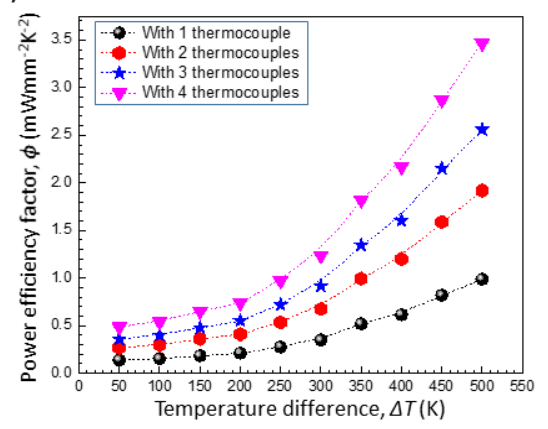

Figure 3. Simulation results showing (a) 3D model of a thermocouple with generated thermoelectric voltage. Enhancement of (b) thermoelectric voltage, (c) Output power, and (d) thermoelectric power efficiency factor while enhancing the number of thermocouples

\section{CONCLUSION}

This paper proposed the modelling and simulation of a $\pi$-shaped $\mathrm{Mg}_{3} \mathrm{Sb}_{2}$ based TEG. The TE performance has been evaluated and analyzed by analytic modelling and finite element simulation. The heat distribution through the $p$-type and $n$-type $\mathrm{Mg}_{3} \mathrm{Sb}_{2}$ based TE legs have been evaluated and it has found that the heat distribution is nearly linear and higher through the $n$-type leg and slightly deviated and lower in $p$-type leg due to high $k$ value for $n$-type than the $p$-type leg. Besides, enhanced $V_{T E}$ value is obtained at high $\Delta T$ with an enhanced number of thermocouples. Moreover, the maximum $P_{\text {out }}$ and $\phi$ is obtained with an enhanced number of thermocouples and due to the low $R_{T E}$ value of both the $\mathrm{Mg}_{3} \mathrm{Sb}_{2}$ based TE legs. The result shows that the developed TEG device has the potential to generate $8.89 \mathrm{~mW}$ and $3.47 \mu \mathrm{Wmm}^{-2} \mathrm{~K}^{-2}$ class maximum output power and maximum output power efficiency, respectively at $\leq 800 \mathrm{~K}$. The analysis performed in this research can be a base to generate power from various sources i.e. from the car engine to human body heat. Thus, most of the research on $\pi$-shaped $\mathrm{Mg}_{3} \mathrm{Sb}_{2}$ based TEG could be seen in on-board power generation devices in the near future. 


\section{ACKNOWLEDGEMENTS}

This work was supported in part by Universiti Teknologi Malaysia and Universiti Malaysia Pahang through Collaborative Research Grant (CRG 05G35 \& CRG 05G02) and the Ministry of Education Malaysia through Fundamental Research under Grant (FRGS 5F081).

\section{REFERENCES}

[1] P. Yu, et al., "Design and fabrication of silicon nanowires towards efficient solar cells," Nano Today, vol. 11, no. 6, pp. 704-737, 2016.

[2] Tan, Jian Ding, et al. "An electromagnetism-like mechanism algorithm approach for photovoltaic system optimization," Indonesian Journal of Electrical Engineering and Computer Science, 12.1, pp. 333-340, 2018.

[3] Chen, Chai Phing, et al. "A heat waste recovery system via thermoelectric generator," Indonesian Journal of Electrical Engineering and Computer Science, vol. 16, no. 2, pp. 586-590, 2019.

[4] Baatar, Nyambayar, and Shiho Kim. "A thermoelectric generator replacing radiator for internal combustion engine vehicles," Telkomnika, vol. 9, no. 3, pp. 523, 2011.

[5] Hasan, M.N., et al. "Inorganic thermoelectric materials: A review," International Journal of Energy Research, 2020; 1-53. https://doi.org/10.1002/er.5313

[6] A. Abdul Tahrim, et al., "Characterization of heat flow in silicon nanowire arrays for efficient thermoelectric power harvesting," Experimental Heat Transfer, vol. 31, no. 6, pp. 470-481, 2018.

[7] K. V. Selvan, et al., "Methodological reviews and analyses on the emerging research trends and progresses of thermoelectric generators," International Journal of Energy Research, vol. 43, no. 1, pp. 113-140, 2019.

[8] K. V. Selvan, et al., "Micro-scale energy harvesting devices: Review of methodological performances in the last decade," Renewable and Sustainable Energy Reviews, vol. 54, pp. 1035-1047, 2016.

[9] H. Lee, et al., "A graphene-based electrochemical device with thermoresponsive microneedles for diabetes monitoring and therapy," Nature Nanotechnology, vol. 11, pp. 566- 572, 2016.

[10] T. Torfs, et al., "Wearable autonomous wireless electro-encephalography system fully powered by human body heat," IEEE Sensors, pp. 1269-1272, 2008.

[11] Y. Wang, et al., "Wearable thermoelectric generator to harvest body heat for powering a miniaturized accelerometer," Applied Energy, vol. 215, pp. 690-698, 2018.

[12] L. Ortega, et al., "Self-powered smart patch for sweat conductivity monitoring," Microsystems \& Nanoengineering, vol. 5, no. 1, pp. 3, 2019.

[13] S. Han, et al., "Thermoelectric Polymer Aerogels for Pressure-Temperature Sensing Applications," Advanced Functional Materials, vol. 27, no. 44, pp. 1703549, 2017.

[14] D. Y. Choi, et al., "Highly Stretchable, Hysteresis-Free Ionic Liquid-Based Strain Sensor for Precise Human Motion Monitoring," ACS Applied Materials \& Interfaces, vol. 9, no. 2, pp. 1770-1780, 2017.

[15] B. Chen, et al., "Flexible thermoelectric generators with inkjet-printed bismuth telluride nanowires and liquid metal contacts," Nanoscale, vol. 11, no. 12, pp. 5222-5230, 2019.

[16] A. Nour Eddine, et al., "Modeling and Simulation of a Thermoelectric Generator Using Bismuth Telluride for Waste Heat Recovery in Automotive Diesel Engines," Journal of Electronic Materials, vol. 48, no. 4, pp. 2036-2045, 2019.

[17] D. Kong, et al., "High-performance flexible $\mathrm{Bi}_{2} \mathrm{Te}_{3}$ films based wearable thermoelectric generator for energy harvesting," Energy, vol. 175, pp. 292-299, 2019.

[18] D. Mei, et al., "Numerical modeling and optimization of the segmented PbTe-BiTe-based thermoelectric leg," Journal of Applied Physics, vol. 120, no. 12, p. 124503, 2016.

[19] X. Wang, et al., "Geometric structural design for lead tellurium thermoelectric power generation application," Renewable Energy, vol. 141, pp. 88-95, 2019.

[20] S. Song, et al., "Joint effect of magnesium and yttrium on enhancing thermoelectric properties of $n$-type Zintl $\mathrm{Mg}_{3+}$ ${ }_{\delta} \mathrm{Y}_{0.02} \mathrm{Sb}_{1.5} \mathrm{Bi}_{0.5}, "$ Materials Today Physics, vol. 8, pp. 25-33, 2019.

[21] A. Bhardwaj, et al., "Significantly enhanced thermoelectric figure of merit of $p$-type $\mathrm{Mg}_{3} \mathrm{Sb}_{2}$-based Zintl phase compounds via nanostructuring and employing high energy mechanical milling coupled with spark plasma sintering," Journal of Materials Chemistry A, vol. 3, no. 20, pp. 10777-10786, 2015.

[22] J. Shuai, et al., "Significant role of $\mathrm{Mg}$ stoichiometry in designing high thermoelectric performance for $\mathrm{Mg}_{3}(\mathrm{Sb}$, Bi) $)_{2}$-based $n$-type zintls," Journal of the American Chemical Society, vol. 140, no. 5, pp. 1910-1915, 2018.

[23] X. Chen, et al., "Extraordinary thermoelectric performance in $n$-type manganese doped $\mathrm{Mg}_{3} \mathrm{Sb}_{2}$ Zintl: High band degeneracy, tuned carrier scattering mechanism and hierarchical microstructure," Nano Energy, vol. 52, pp. 246-255, 2018.

[24] K. V. Selvan, et al., "State-of-the-Art Reviews and Analyses of Emerging Research Findings and Achievements of Thermoelectric Materials over the Past Years," Journal of Electronic Materials, vol. 48, no. 2, pp. 745-777, 2019.

[25] T. Zhu, et al., "Compromise and synergy in high-efficiency thermoelectric materials," Advanced materials, vol. 29 , no. 14, pp. 1605884, 2017.

[26] G. Pennelli, "Review of nanostructured devices for thermoelectric applications," Beilstein journal of nanotechnology, vol. 5, pp. 1268- 1284, 2014.

[27] L. E. Bell, "Cooling, heating, generating power, and recovering waste heat with thermoelectric systems," Science, vol. 321 , no. 5895, pp. 1457-1461, 2008. 
[28] K. V. Selvan, et al., "Copper-Nickel and Copper-Cobalt Thermoelectric Generators: Power-Generating Optimization through Structural Geometrics," IEEE Transactions on Electron Devices, vol. 65, no. 8, pp. 3394-3400, 2018.

[29] M. Wahbah, et al., "Characterization of human body-based thermal and vibration energy harvesting for wearable devices," IEEE Journal on Emerging and Selected Topics in Circuits and Systems, vol. 4, no. 3, pp. 354-363, 2014.

[30] K. V. Selvan, et al., "Copper-Cobalt Thermoelectric Generators: Power Improvement Through Optimized Thickness and Sandwiched Planar Structure," IEEE Transactions on Electron Devices, vol. 66, no. 8, pp. 3459-3465, 2019.

[31] S. Zhou, et al., "Multiscale modeling of Thermoelectric Generators for conversion performance enhancement," International Journal of Heat and Mass Transfer, vol. 81, pp. 639-645, 2015.

[32] B. Jang, et al., "Optimal design for micro-thermoelectric generators using finite element analysis," Microelectronic Engineering, vol. 88, no. 5, pp. 775-778, 2011.

[33] H. Sun, et al., "Geometric optimization of two-stage thermoelectric generator using genetic algorithms and thermodynamic analysis," Energy, vol. 171, pp. 37-48, 2019.

\section{BIOGRAPHIES OF AUTHORS}

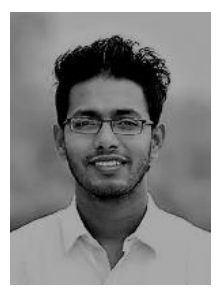

Md. Nazibul Hasan received B. Eng. degree in Electronics and Telecommunication Engineering from Gauhati University, India in 2013. He received the $M$. Tech. degree in Mobile Communication and Computing from National Institute of Technology, Arunachal Pradesh, India in 2016. He is currently pursuing his Ph.D. degree in Electrical Engineering at Universiti Teknologi Malaysia. His research interests include Energy Harvesting Devices, MEMS, and NEMS.

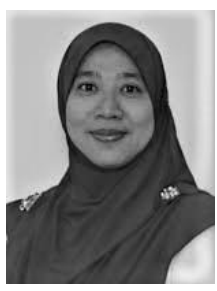

Herlina Abdul Rahim received a B.Eng in Electrical Engineering (Control and Instrumentation) and M.Sc in Electrical Engineering from Universiti Teknologi Malaysia in 1998 and 2000, respectively. She received her Ph.D in Electrical Engineering from Universiti Teknologi MARA, in 2009. At present she is an Associate Professor at Faculty of Electrical Engineering, Universiti Teknologi Malaysia. She is currently a Deputy Director (Corporate Management) at Research Management Centre in UTM. Her current research interests are sensor technology and artificial intelligent system. She actively involved in R\&D and to date, she has filed 72 IPR including patent filings and copyrights. She has won many medals for her inventions and innovations at international and local research exhibition. She has also published more than 200 research articles in international journals, proceedings, book chapters and research monographs. She is a senior member of the IEEE, a Professional Engineer of Board of Engineers Malaysia (BEM), a corporate member of the Institute of Engineers Malaysia (IEM) and Chartered Engineer, the Institute of Engineering and Technology.

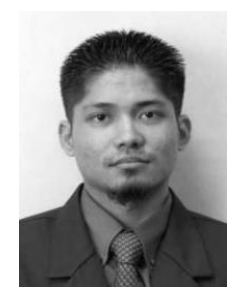

Mohd Ashraf Ahmad received his first degree in B.Eng Electrical Mechatronics and Master Degree in M.Eng Mechatronics and Automatic Control from University of Technology Malaysia (UTM) in 2006 and 2008, respectively. In 2015, he received a Ph.D in Informatics (Systems Science) from Kyoto University. Currently, he is a senior lecturer in the Faculty of Electrical and Electronics Engineering Technology, University Malaysia Pahang (UMP). His current research interests are model-free control, control of Mechatronic systems, nonlinear system identification and vibration control. He has been serving as Associate Editor for the International Journal of Electrical and Computer Engineering since 2016, Applications of Modelling and Simulation since 2017, and Journal of Future Robot Life since 2019.

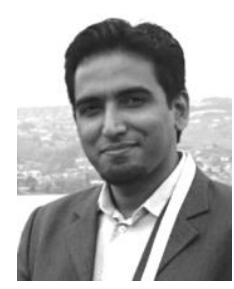

Mohamed Sultan Mohamed Ali received the B.Eng. and M.Eng. degrees in electrical engineering from Universiti Teknologi Malaysia, Skudai, Johor, Malaysia, in 2006 and 2008, respectively, and the $\mathrm{Ph} . \mathrm{D}$. degree in electrical and computer engineering from the Department of Electrical and Computer Engineering, The University of British Columbia, Vancouver, BC, Canada, in 2012. From 2001 to 2007, he held various engineering positions at Flextronics International Ltd. and Jabil Circuit, Inc. He is currently an Associate Professor in the Faculty of Electrical Engineering, Universiti Teknologi Malaysia. Dr. Mohamed Sultan serves as a consultant for Flextronics Malaysia in the failure analysis division and is actively involved in many industrial projects. He was awarded Chartered Engineer (C.Eng.) and Professional Engineer (P.Eng.) by United Kingdom Engineering Council and Board of Engineers Malaysia, respectively. Dr. Mohamed Sultan is also a Senior Member of IEEE (SMIEEE). Dr. Mohamed Sultan has published over 100 technical papers in MEMS and related fields. He has also served as the editor of 5 books in Mechatronics. His research interests are in the areas of MEMS, nanotechnology, and also in micro/nanofabrication technologies, including wireless microdevices, integration of microstructures, and microrobotics. 